\title{
Factors Associated with the Hospitalization of Low-risk Patients with Community-acquired Pneumonia in a Cluster-Randomized Trial
}

\author{
Jose Labarere, MD, PhD, ${ }^{1,2}$ Roslyn A. Stone, PhD, ${ }^{1,3}$ D. Scott Obrosky, MSc, ${ }^{1}$ \\ Donald M. Yealy, MD, ${ }^{4}$ Thomas P. Meehan, MD, MPH, ${ }^{5,6}$ Thomas E. Auble, PhD, ${ }^{4}$ \\ Jonathan M. Fine, MD, ${ }^{6,7}$ Louis G. Graff, MD, $, 8,8,9$ Michael J. Fine, MD, MSc ${ }^{1,10}$ \\ 'VA Center for Health Equity Research and Promotion, VA Pittsburgh Healthcare System, Pittsburgh, PA, USA; ${ }^{2}$ ThEMAS TIMC-IMAG UMR \\ CNRS 5525 UJF, Grenoble University Hospital, Grenoble, France; ${ }^{3}$ Department of Biostatistics, University of Pittsburgh Graduate School of \\ Public Health, Pittsburgh, PA, USA; ${ }^{4}$ Department of Emergency Medicine, University of Pittsburgh, Pittsburgh, PA, USA; ${ }^{5}$ Department of \\ Medicine, Yale University School of Medicine, New Haven, CT, USA; ${ }^{6}$ Qualidigm, Middletown, CT, USA; ${ }^{7}$ Section of Pulmonary and Critical \\ Care Medicine, Norwalk Hospital, Norwalk, CT, USA; ${ }^{8}$ Department of Emergency Medicine, University of Connecticut School of Medicine, \\ Farmington, CT, USA; ${ }^{2}$ Department of Emergency Medicine, New Britain General Hospital, New Britain, CT, USA; ${ }^{0}$ Division of General \\ Internal Medicine, Department of Medicine, University of Pittsburgh, Pittsburgh, PA, USA.
}

\begin{abstract}
BACKGROUND: Many low-risk patients with pneumonia are hospitalized despite recommendations to treat such patients in the outpatient setting.
\end{abstract}

OBJECTIVE: To identify the factors associated with the hospitalization of low-risk patients with pneumonia.

METHODS: We analyzed data collected by retrospective chart review for 1,889 low-risk patients (Pneumonia Severity Index [PSI] risk classes I to III without evidence of arterial oxygen desaturation) enrolled in a cluster-randomized trial conducted in 32 emergency departments.

RESULTS: Overall, 845 (44.7\%) of all low-risk patients were treated as inpatients. Factors independently associated with an increased odds of hospitalization included PSI risk classes II and III, the presence of medical or psychosocial contraindications to outpatient treatment, comorbid conditions that were not contained in the PSI (cognitive impairment, history of coronary artery disease, diabetes mellitus, or pulmonary disease), multilobar radiographic infiltrates, and home therapy with oxygen, corticosteroids, or antibiotics before presentation. While $32.8 \%$ of low-risk inpatients had a contraindication to outpatient treatment and $47.1 \%$ had one or more preexisting treatments, comorbid conditions, or radiographic abnormalities not contained in the PSI, $20.1 \%$ had no identifiable risk factors for hospitalization other than PSI risk class II or III.

CONCLUSIONS: Hospital admission appears justified for one-third of low-risk inpatients based upon the presence of one or more contraindications to outpatient treatment. At least one-fifth of low-risk inpatients did not have a contraindication to outpatient treatment or an identifiable risk factor for hospitalization, suggesting that treatment of a larger proportion of such low-risk patients in the outpatient setting could be achieved without adversely affecting patient outcomes.

KEY WORDS: pneumonia; community-acquired infections; patient admission; risk factors; emergency service, hospital.

DOI: $10.1111 /$ j.1525-1497.2006.00510.x

J GEN INTERN MED 2006; 21:745-752.

$\mathrm{C}$ ommunity-acquired pneumonia (designated pneumonia) causes 4 million episodes of illness and more than $1 \mathrm{mil}$ lion hospital admissions in the United States each year. ${ }^{1}$ The magnitude of resource use for pneumonia is directly related to inpatient treatment, which costs approximately 20 times as

Address correspondence and requests for reprints to Dr. Fine: VA Center for Health Equity Research and Promotion, VA Pittsburgh Healthcare System, University Drive C, Building 28, 1A102, Pittsburgh, PA 15240 (e-mail: Michael.Fine@med.va.gov). much as outpatient care. ${ }^{2}$ Owing to the large cost differences associated with the site of treatment, increasing outpatient management of low-risk patients is one approach to improve the cost efficiency of care for this illness. Other research has demonstrated that physicians may overestimate the severity of illness in low-risk patients with pneumonia, leading to potentially unnecessary hospitalizations rather than the outpatient treatment that is often preferred by patients. ${ }^{3,4}$

The Pneumonia Severity Index (PSI) is a validated prediction rule for prognosis that identifies patients with pneumonia at low risk for 30-day mortality and other adverse medical outcomes. ${ }^{5}$ Three previous studies, including the recently completed Emergency Department Community-Acquired Pneumonia (EDCAP) trial, demonstrated the effectiveness and safety of using the PSI to reduce the hospitalization of low-risk patients. ${ }^{6-8}$ Despite the implementation of interventions to increase the proportion of low-risk patients treated in the outpatient setting in these trials, the proportion of low-risk patients hospitalized ranged from $31 \%$ to $62.5 \%$. Limited data document the risk factors for the hospitalization of low-risk patients with pneumonia. ${ }^{9,10}$ Identifying these factors and understanding whether hospitalized low-risk patients have one or more of these factors at presentation could lead to further improvements in the efficiency of hospital use among patients with pneumonia and improved interventions to guide the initial site of treatment for such patients.

The aim of the present study was to identify the patient, physician, and emergency department characteristics associated with the hospitalization of low-risk patients enrolled in the EDCAP trial. More specifically, we focused on baseline patient characteristics that are not prognostic factors contained in the PSI, such as preexisting medical treatments, coexisting comorbid illnesses, or medical or psychosocial contraindications to outpatient care.

\section{PATIENTS AND METHODS}

\section{Study Design}

The EDCAP trial is a 32 -site cluster-randomized trial designed to compare the effectiveness and safety of 3 emergency de-

Manuscript received November 11, 2005

Initial editorial decision January 24, 2006

Final acceptance March 10, 2006 
partment-level interventions to use the PSI to guide the initial site of treatment of patients with pneumonia. ${ }^{11}$ In this trial, outpatient treatment was recommended for all low-risk patients, defined as those in PSI risk classes I-III with no evidence of arterial oxygen desaturation (oxygen saturation $<90 \%$ or oxygen tension $<60 \mathrm{mmHg}$ ). Inpatient treatment was recommended for all patients with evidence of oxygen desaturation and/or patients in risk classes IV or $\mathrm{V}$ (i.e., higher-risk patients). ${ }^{6,11}$

\section{Study Population}

Physicians from 16 emergency departments in Connecticut and 16 emergency departments in Western Pennsylvania recruited patients 24 hours a day, 7 days a week between January 2001 and December 2001. Eligible patients were 18 years of age or older with a clinical diagnosis of pneumonia and a new radiographic pulmonary infiltrate. Patients were excluded if they were considered to have hospital-acquired pneumonia, pulmonary tuberculosis, immune suppression, positive serology for human immunodeficiency virus, alcoholism with evidence of end-organ damage, illicit drug use within the past 30 days, or social problems incompatible with outpatient treatment, enrollment, or follow-up (e.g., homeless, incarcerated as a prisoner). ${ }^{11}$ Although the trial included recommendations for low-risk and higher-risk patients, the present analysis focuses on the hospital admission decision for lowrisk patients.

\section{Data Collection}

Trained research nurses collected the following baseline data using a medical record review: patient sociodemographics, initial vital signs and mental status, comorbid conditions, medical treatments before presentation, physical examination findings, pertinent laboratory test results, and chest radiography findings from the time of presentation. The PSI risk class was calculated based on the 20 prognostic variables collected retrospectively during the medical record review. For all of the dichotomous variables included in the PSI, missing values were assumed to be normal; this is the same strategy used in the original derivation and validation of the PSI. ${ }^{5}$ Data on comorbid conditions that were not contained in the PSI included cognitive impairment, history of coronary artery disease, pulmonary disease (i.e., obstructive, reactive, or restrictive lung disease), and diabetes mellitus. Data on medical treatments before and following presentation included home oxygen therapy, oral or inhaled corticosteroid therapy, antibiotic therapy within 7 days of presentation, and type and route of antibiotic therapy for pneumonia.

Psychosocial and medical absolute contraindications to outpatient treatment of low-risk patients that were explicitly identified in the EDCAP project guideline included the following new conditions or exacerbations of chronic conditions documented as present during the visit at the emergency department: (1) stupor or coma, (2) severe dementia, delirium, psychiatric illness, acute confusion, or disorientation that may affect compliance with oral antibiotic regimen or other outpatient treatments, (3) intractable vomiting or inability to take oral antibiotics, (4) frailty or serious neuromuscular disorder with inability to conduct activities of daily living at home, (5) known or suspected severe concomitant illness requiring hospitalization (i.e., unstable angina, acute myocardial infarction, life-threatening ventricular arythmia, cardiopulmonary arrest, gastrointestinal bleeding with volume depletion, acute asthma or exacerbation of obstructive pulmonary disease with severe bronchospasm treated with intravenous steroids, heart failure or pulmonary edema treated with intravenous diuretics, diabetic ketoacidosis or hyperosmolar coma, acute renal failure, and venous thromboembolic disease), (6) known or suspected concomitant suppurative infection (i.e., empyema, septic arthritis, bacterial meningitis, endocarditis, peritonitis, brain abscess, pulmonary abscess, or osteomyelitis), (7) severe abnomality in one or more vital signs (i.e., pulse $>150 /$ minute, respiratory rate $>40 /$ minute, or systolic blood pressure $<60 \mathrm{~mm} \mathrm{Hg}$ ), or laboratory values (i.e., hematocrit $<24 \%$, serum sodium $<120 \mathrm{mEq} / \mathrm{L}$, serum glucose $>500 \mathrm{mg} / \mathrm{dL}$, or arterial $\mathrm{pH}<7.25$ ) documented at any time in the emergency department. ${ }^{11}$

We also collected the characteristics of participating emergency departments, and the demographic and professional characteristics of all physicians in participating emergency departments.

\section{Site of Treatment}

We defined inpatient treatment as hospital admission, transfer from an emergency department to an inpatient hospital observation unit, or admission to an emergency department observation unit with discharge to any setting more than 24 hours after initial presentation. Conversely, we defined outpatient treatment as discharge from the emergency department to any outpatient setting or discharge from an emergency department observation unit within 24 hours of initial presentation.

\section{Statistical Analysis}

We used multilevel logistic regression to analyze inpatient treatment with the 3 levels defined by patient, physician, and emergency department. ${ }^{12}$ We used the Metropolis-Hastings algorithm (a Markov Chain Monte Carlo method) in MLwiN statistical software (Centre for multilevel modelling, London, UK) to account for the cross-classification of the 21 physicians who enrolled patients at more than one study site. ${ }^{13}$ For each predictor considered, we present the odds ratio (OR) of inpatient treatment and associated 95\% confidence interval (CI), adjusted for the EDCAP trial intervention arm. In multivariable analysis, we developed a parsimonious model by removing variables from the full main effects model using a backward approach, with a cutoff of $P=.05$. Independent variables entered into the model included PSI risk class, presence of one or more contraindications to outpatient treatment, presence of one or more comorbid conditions not in the PSI, home oxygen therapy, corticosteroid therapy, antibiotic therapy within 7 days, multilobar radiographic infiltrates, bilateral radiographic infiltrates, presentation at night or on weekend, medical insurance status, antibiotic allergy or intolerance, and nonwhite race. In order to account for the study design of the EDCAP trial, we forced the study intervention arm and the emergency department stratification variables (i.e., teaching status, state, and annual volume) into the multivariable model. Two-sided $P$ values of less than .05 were considered statistically significant. 


\section{RESULTS}

The EDCAP trial enrolled 3,615 (80.2\%) of 4,506 eligible patients. Non-enrolled eligible patients were older and more likely to reside in a nursing home than enrolled patients. We excluded 414 enrolled patients because of the discovery of an exclusion criterion after enrollment or protocol or consent violations (Fig. 1). After 1,312 higher-risk patients were excluded, the study population included 1,889 low-risk patients, with a median of 59 low-risk patients enrolled per emergency department (interquartile range $[\mathrm{IQR}]=48$ to 69 ).

\section{Patient Characteristics}

The median age of all low-risk patients was 54 years $(\mathrm{IQR}=38$ to 71$)$; 862 (45.6\%) were male, 310 (16.4\%) nonwhite, 193 (10.2\%) uninsured, and $845(44.7 \%)$ treated as inpatients. At presentation, 670 patients (35.5\%) were in PSI risk class I, 702 $(37.2 \%)$ in class II, and 517 (27.4\%) in class III. Overall, 350

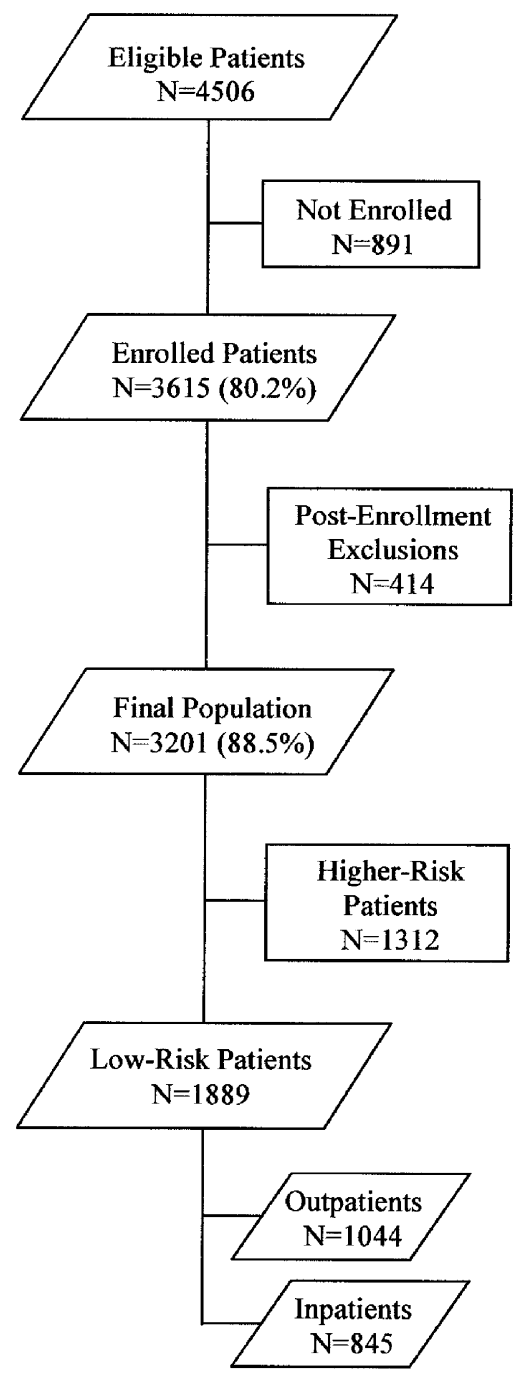

FIGURE 1. Patient enrollment. Low-risk patients were in Pneumonia Severity Index (PSI) risk classes I to III with no evidence of arterial oxygen desaturation (oxygen saturation $<90 \%$ or oxygen tension $<60 \mathrm{mmHg}$ ), and higher-risk patients comprised all patients with evidence of oxygen desaturation and/or patients in risk classes IV or V. patients (18.5\%), including $73(7.0 \%)$ outpatients and 277 $(32.8 \%)$ inpatients, had one or more predefined contraindications to outpatient care. The majority of low-risk inpatients $(n=736 ; 87.1 \%)$ and minority of low-risk outpatients $(n=308$; $29.5 \%$ ) received one or more doses of intravenous antibiotic for pneumonia.

\section{Univariate Comparisons of Low-risk Inpatients and Outpatients}

As shown in Table 1, PSI risk class was associated with inpatient treatment among low-risk patients. Of the 20 individual prognostic factors that constitute the PSI, 8 were associated with significantly increased odds of inpatient treatment (age, congestive heart failure, cerebrovascular disease, renal disease, respiratory rate $\geq 30$ per minute, pulse $\geq 125$ per minute, elevated BUN, and pleural effusion).

Non-white race, Medicaid insurance or no health insurance, and presentation at night or on a weekend were associated with decreased odds of inpatient treatment (Table 2). In contrast, having one or more contraindications to outpatient treatment; one or more comorbid conditions, laboratory or radiographic abnormalities that were not prognostic factors contained in the PSI; or therapy with oxygen, oral or inhaled corticosteroids, or antibiotics before presentation were all associated with an increased odds of inpatient treatment (Table 2). No physician or emergency department characteristics other than intervention arm were associated with the initial site of treatment (data not shown).

\section{Factors Independently Associated with Inpatient Treatment of Low-Risk Patients}

In multivariable analysis, the factors independently associated with increased odds of inpatient treatment were PSI risk class, one or more contraindications to outpatient treatment, one or more comorbid conditions not contained in the PSI, therapy with oxygen, corticosteroids, or antibiotics before presentation, and presence of multilobar radiographic infiltrates (Table 3). Two factors independently associated with decreased odds of inpatient treatment were Medicaid insurance and presentation at night or on a weekend. Use of a prospective assessment of PSI risk class did not affect the patient characteristics that were independently associated with the site of treatment or the magnitude of their effect.

Overall, patients with one or more contraindications to outpatient treatment accounted for $32.8 \%$ of low-risk inpatients, patients with one or more comorbid conditions that were not contained in the PSI, multilobar radiographic infiltrates, or treatment with oxygen, corticosteroid, or antibiotics before presentation accounted for $47.1 \%$ of low-risk inpatients, and patients with no identifiable risk factors for hospitalization other than PSI risk class accounted for $20.1 \%$ of low-risk inpatients (Table 4). Of the 170 low-risk inpatients who had no factor for hospitalization other than those that determine PSI risk class, $20.6 \%$ were assigned to PSI risk class I, $46.5 \%$ to risk class II, and $32.9 \%$ to risk class III.

\section{DISCUSSION}

Outpatient treatment is frequently recommended for low-risk patients with pneumonia. ${ }^{14-16}$ Patients assigned to PSI risk classes I to III are at low risk for short-term mortality and 
Table 1. Comparison of Variables in the Pneumonia Severity Index Between Low-Risk Outpatients and Inpatients

\begin{tabular}{|c|c|c|c|c|c|c|}
\hline Characteristics & \multicolumn{2}{|c|}{ Outpatients } & \multicolumn{2}{|c|}{ Inpatients } & \multicolumn{2}{|c|}{ Odds Ratio of Inpatient Treatment $(95 \% \mathrm{Cl})^{*}$} \\
\hline \multicolumn{7}{|l|}{ Pneumonia Severity Index ${ }^{\dagger}$} \\
\hline Class II & 365 & (35.0) & 337 & (39.9) & 5.00 & (3.70 to 6.67 ) \\
\hline Class III & 129 & (12.3) & 388 & $(45.9)$ & 16.67 & (12.50 to 25.00$)$ \\
\hline \multicolumn{7}{|l|}{ Demographic factors } \\
\hline Nursing home resident & 0 & $(0.0)$ & 6 & $(0.7)$ & - & - \\
\hline \multicolumn{7}{|l|}{ Comorbid conditions } \\
\hline Neoplastic disease & 4 & $(0.4)$ & 5 & $(0.6)$ & 1.49 & - $^{*}$ \\
\hline Liver disease & 10 & (1.0) & 5 & (0.6) & 0.62 & (0.19 to 2.08$)$ \\
\hline Congestive heart failure & 16 & (1.5) & 63 & (7.5) & 5.88 & (3.33 to 11.11 ) \\
\hline Cerebrovascular disease & 24 & (2.3) & 42 & (5.0) & 2.50 & (1.43 to 4.54$)$ \\
\hline Renal disease & 5 & $(0.5)$ & 11 & (1.3) & 3.33 & (1.00 to 11.11 ) \\
\hline Pulse $\geq 125$ per minute & $64 / 1,042$ & (6.1) & $104 / 843$ & (12.3) & 2.32 & (1.61 to 3.33$)$ \\
\hline \multicolumn{7}{|l|}{ Laboratory and X-ray findings ${ }^{\S}$} \\
\hline Arterial $\mathrm{pH}<7.35$ & $0 / 38$ & $(0.0)$ & $3 / 160$ & (1.9) & - & $-^{*}$ \\
\hline $\mathrm{BUN}>30 \mathrm{mg} / \mathrm{dL}$ & $6 / 432$ & (1.4) & $27 / 813$ & (3.3) & 2.78 & (1.04 to 7.14$)$ \\
\hline Sodium < $130 \mathrm{mEq} / \mathrm{L}$ & $8 / 438$ & (1.8) & $20 / 813$ & (2.5) & 1.35 & (0.54 to 3.33$)$ \\
\hline Glucose $\geq 250 \mathrm{mg} / \mathrm{dL}$ & $17 / 437$ & (3.9) & $38 / 803$ & (4.7) & 1.30 & (0.69 to 2.44$)$ \\
\hline Hematocrit $<30 \%$ & $11 / 526$ & (2.1) & $24 / 835$ & (2.9) & 1.25 & (0.56 to 2.78 ) \\
\hline Radiographic pleural effusion & $44 / 1,041$ & $(4.2)$ & $81 / 837$ & (9.7) & 2.63 & (1.69 to 4.00$)$ \\
\hline
\end{tabular}

*Odds ratios were adjusted for intervention arm and were estimated using multilevel logistic regression with the 3 levels defined by patient, physician, and emergency department. Confidence intervals (CI) were not estimated if less than 5 outpatients or inpatients had the characteristic.

${ }^{\dagger}$ Missing values were assumed to be normal when calculating the Pneumonia Severity Index, as performed in the original derivation and validation of this prognostic rule ${ }^{5}$.

${ }^{\ddagger}$ Values for physical examination findings were missing for respiratory rate $(\mathrm{n}=11)$, systolic blood pressure $(\mathrm{n}=8)$, temperature $(\mathrm{n}=18)$, and pulse rate $(\mathrm{n}=4)$.

${ }^{\S}$ Values for laboratory and $x$-ray findings were missing for arterial $p H(\mathrm{n}=1,691)$, blood urea nitrogen (BUN) $(\mathrm{n}=644)$, sodium (n=638), glucose $(\mathrm{n}=649)$, hematocrit $(\mathrm{n}=528)$, and radiographic pleural effusion $(\mathrm{n}=11)$.

adverse medical outcomes based on the derivation and validation cohorts of the PSI. ${ }^{5}$ Outpatient treatment is often preferred by low-risk patients ${ }^{4}$ and costs substantially less than inpatient treatment. $^{2}$ Yet, $44.7 \%$ of low-risk patients enrolled in the EDCAP trial were hospitalized despite the implementation of low, moderate, and high-intensity interventions to increase treatment in the outpatient setting. This observation highlights the importance of physician judgment in the initial site of treatment decision for low-risk patients with pneumonia.

In this study, we identified the patient characteristics that are independently associated with the initial site of treatment of low-risk patients enrolled in the EDCAP trial. In addition to PSI risk classes II and III, patient characteristics associated with the hospitalization of low-risk patients were the presence of medical or psychosocial contraindications to outpatient treatment, comorbid conditions not contained in the PSI, multilobar radiographic infiltrates, and therapy with oxygen, corticosteroids, or antibiotics before admission. Two patient-level factors, Medicaid insurance and presentation at night or on a weekend, were negatively associated with inpatient treatment in our multivariable analysis. A previous study found a similar negative association between Medicaid insurance and inpatient treatment ${ }^{10}$; however, other studies have identified low socioeconomic status as a major reason for hospital admission of low-risk patients with pneumonia. ${ }^{17-19}$ One potential expla- nation for this discrepancy is that some characteristics associated with low socioeconomic status, such as homelessness, pulmonary tuberculosis, positive human immunodeficiency virus serology, alcoholism, and illicit drug use, were used as exclusion criteria in our study. Indeed, Stelianides et al. ${ }^{20}$ reported that patients with low socioeconomic status did not have more severe presentation or worse outcomes than other patients, after adjusting for these potential confounding factors. That patients presenting at night or on weekends were less likely to be treated as inpatients was consistent with the study of Halm et al. ${ }^{10}$ and might be explained by the fact that these patients were more likely to be workers. The relative frequency of hospital admissions related to severe alcoholism or social problems incompatible with outpatient treatment, which often represent compelling reasons for inpatient care, could not be assessed in our study population owing to the exclusion criteria of the EDCAP trial.

Three distinct groups of hospitalized low-risk patients can be identified based upon the risk factors for hospitalization identified in this study. The first group consisted of patients with medical or psychosocial contraindications to outpatient treatment. Although hospital admission of these patients was likely to reflect physician compliance with explicit criteria in the EDCAP project guideline that identified contraindications to outpatient treatment, ${ }^{11}$ it is important to notice that this 
Table 2. Comparison of Variables not in the Pneumonia Severity Index between Low-Risk Outpatients and Inpatients

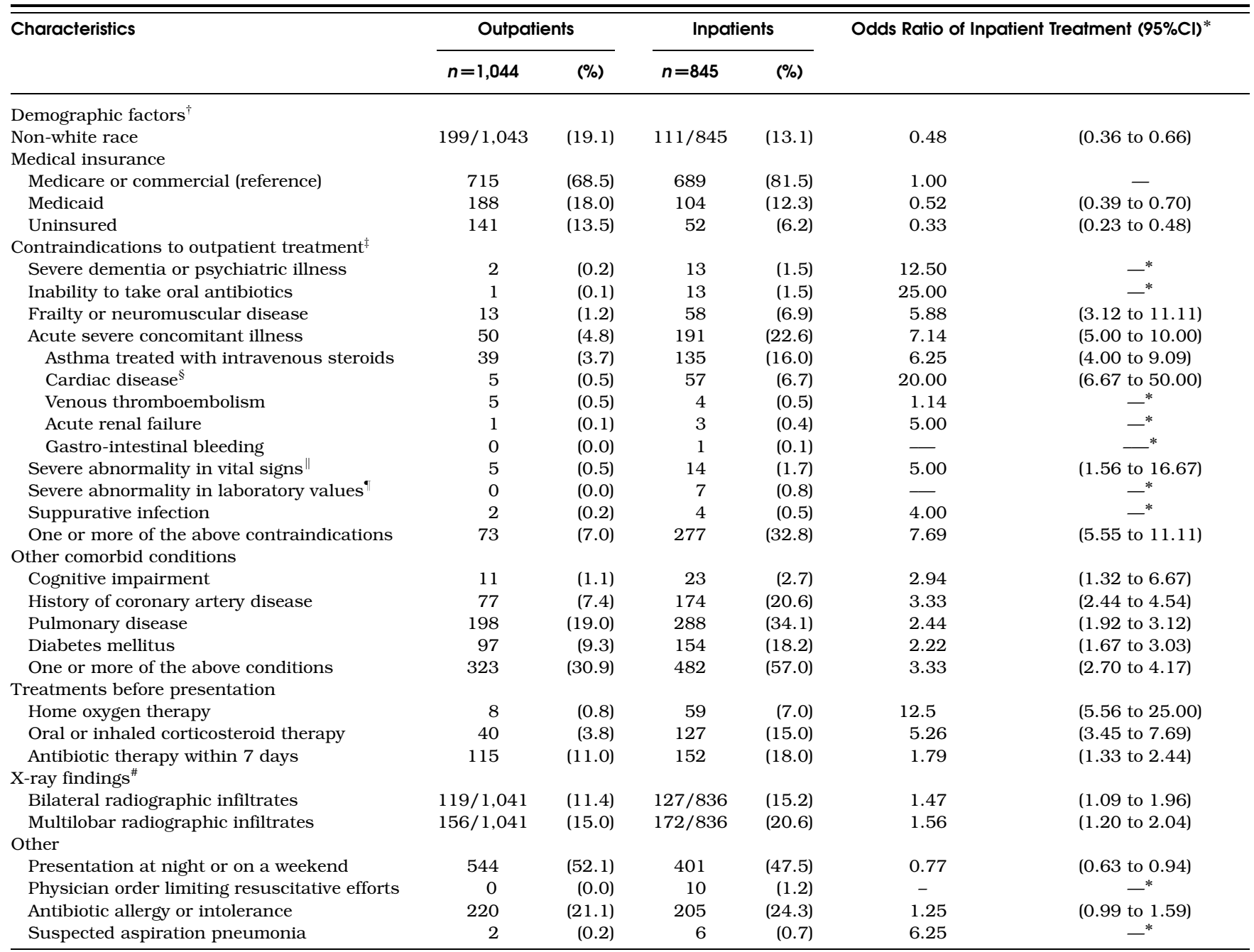

* Odds ratios were adjusted for intervention arm and were estimated using multilevel logistic regression with the 3 levels defined by patient, physician, and emergency department. Confidence intervals (CI) were not estimated if less than 5 outpatients or inpatients had the characteristic.

${ }^{\dagger}$ Race was missing for 1 patient.

${ }^{\ddagger}$ No patients had stupor, coma, or diabetic ketoacidosis.

${ }^{\S}$ Cardiac disease included unstable angina, acute myocardial infarction, heart failure or pulmonary edema treated with intravenous diuretics, and life-threatening arrhythmia or cardiac arrest.

"A severe abnormality in one or more vital signs consisted of: pulse $>150$ per minute, respiratory rate $>40$ per minute, or systolic blood pressure $<60 \mathrm{mmHg}$

"A severe abnormality in 1 or more laboratory values consisted of: hematocrit $<24 \%$, serum sodium $<120 \mathrm{meq} / \mathrm{L}$, serum glucose $>500 \mathrm{mg} / \mathrm{dL}$, or arterial $p H<7.25$.

${ }^{\#}$ Values for $x$-ray findings were missing for bilateral radiographic infiltrates $(\mathrm{n}=12)$ and multilobar radiographic infiltrates $(\mathrm{n}=12)$.

group of patients accounted for only one-third of low-risk patients who were hospitalized.

The second group of hospitalized low-risk inpatients were those with preexisting treatments, radiographic abnormalities, or comorbid conditions not contained in the PSI. Among these medical factors associated with an increased odds of inpatient treatment, pulmonary disease, diabetes mellitus, history of coronary artery disease, ${ }^{3,10}$ multilobar radiographic infiltrates, ${ }^{3,10,18,19,21}$ and therapy with antibiotics before admission $^{10,18,19,21}$ were previously identified as reasons for hospital admission of low-risk patients. Although not previously identified in the literature, we also found that home oxygen therapy and corticosteroid therapy were strong independent predictors of hospital admission of low-risk patients. Despite the lack of published data consistently supporting an increased risk of mortality or other adverse medical outcomes for these factors, together, these characteristics accounted for $47.1 \%$ of hospitalizations for low-risk patients in our study. Pulmonary disease, diabetes mellitus, and history of coronary artery disease were not incorporated into the original PSI because they were not found to be independent predictors of mortality. ${ }^{5}$ Additionally, no consistent association between pulmonary disease and short-term mortality following an episode of pneumonia has been documented in the medical literature. ${ }^{22}$ Although significant univariate associations between short-term mortality and diabetes mellitus, ${ }^{22,23}$ history of coronary artery disease, ${ }^{22}$ or presence of multilobar radiographic infiltrates ${ }^{22,24}$ exist, there is no evidence that these factors are independent 
Table 3. Independent Associations of Patient and Emergency Department Characteristics with Inpatient Treatment of Low-Risk Patients

\begin{tabular}{|c|c|c|}
\hline \multirow{2}{*}{$\begin{array}{l}\text { Characteristics* }^{*} \\
\text { Emergency department characteristics }(n=32)\end{array}$} & \multicolumn{2}{|c|}{ Adjusted Odds Ratio of Inpatient Treatment $(95 \% \mathrm{Cl})^{\dagger}$} \\
\hline & & \\
\hline \multicolumn{3}{|l|}{ Intervention arm } \\
\hline Low intensity $(n=8)$ (reference) & 1.00 & - \\
\hline Moderate intensity $(n=12)$ & 0.37 & $(0.20$ to 0.71$)$ \\
\hline High intensity $(n=12)$ & 0.58 & $(0.30$ to 1.09$)$ \\
\hline Location in Connecticut $(n=16)$ & 0.67 & $(0.40$ to 1.12$)$ \\
\hline Teaching hospital $(n=16)$ & 1.47 & (0.89 to 2.44$)$ \\
\hline Annual volume $\geq 34,000$ visits $(n=16$ ) & 1.26 & $(0.75$ to 2.17$)$ \\
\hline \multicolumn{3}{|l|}{ Patients characteristics $(n=1,877)$} \\
\hline \multicolumn{3}{|l|}{ Pneumonia Severity Index } \\
\hline Class I (reference) & 1.00 & - \\
\hline Class II & 4.00 & (2.94 to 5.26$)$ \\
\hline Class III & 12.50 & (8.33 to 16.67$)$ \\
\hline One or more contraindications to outpatient treatment & 5.26 & (3.85 to 7.69$)$ \\
\hline One or more comorbid conditions not in the PSI ${ }^{\ddagger}$ & 1.56 & (1.20 to 2.04$)$ \\
\hline Home oxygen therapy & 3.45 & (1.39 to 8.33$)$ \\
\hline Corticosteroid therapy & 1.92 & $(1.19$ to 3.12$)$ \\
\hline Antibiotic therapy within 7 days & 1.61 & (1.14 to 2.27$)$ \\
\hline Multilobar radiographic infiltrates & 1.79 & (1.32 to 2.43$)$ \\
\hline Presentation at night or on a weekend & 0.76 & (0.60 to 0.98$)$ \\
\hline \multicolumn{3}{|l|}{ Medical insurance } \\
\hline Medicare or commercial (reference) & 1.00 & - \\
\hline Medicaid & 0.63 & $(0.44$ to 0.89$)$ \\
\hline Uninsured & 0.79 & $(0.51$ to 1.22$)$ \\
\hline
\end{tabular}

${ }^{*}$ Twelve patients were excluded from multivariable analysis because of missing values. No physician characteristics were associated with inpatient treatment in multivariable analysis.

Odds ratios were estimated using multilevel logistic regression with the 3 levels defined by patient, physician, and emergency department. CI denotes confidence interval.

${ }^{\ddagger}$ Comorbid conditions not in the PSI included cognitive impairment, history of coronary artery disease, pulmonary disease, and diabetes mellitus.

predictors of mortality after adjusting for other confounders of pneumonia severity.

The third group, patients who had no identifiable factors for hospitalization other than those that determine PSI risk class, accounted for $20.1 \%$ of the low-risk inpatients. While one-third of these patients were in PSI risk class III, the majority (67.1\%) were in PSI risk classes I and II. The reasons for hospitalization of such patients are unclear and may have included patient or family preferences, physician risk aversion, or physician judgment that the patient's severity of illness warranted hospitalization despite their low-risk classification and the absence of medical or psychosocial contraindications to outpatient care.

What are the clinical implications for these 3 groups of low-risk inpatients? Hospital admission appears to be clearly justified for the first group of low-risk inpatients who had one or more delineated contraindications to outpatient treatment.
Although such patients who have a terminal illness or a "no code" status and strong preference for treatment outside the hospital are legitimate exceptions to this rule, the majority of patients in this group would likely benefit from treatment or observation in a hospital setting. In contrast, the appropriateness of hospitalization for the third group of inpatients with no contraindications to outpatient treatment and no discernable risk factor for hospitalization is questionable. In a randomized-controlled trial, Carratala et al. ${ }^{25}$ found that outpatient treatment was as effective and safe as hospitalization in 224 patients in PSI risk classes II to III without unstable comorbid conditions or contraindications to outpatient treatment. It is likely that the majority of these patients could have been safely treated in the outpatient setting. The ideal site of treatment for the second group of patients remains uncertain. Although these patients have identifiable risk factors for hospitalization, none of these factors represent absolute indi-

Table 4. Frequency of Independent Risk Factors for Hospitalization for Low-Risk Outpatients and Inpatients

\begin{tabular}{|c|c|c|c|c|c|}
\hline \multirow{2}{*}{$\begin{array}{l}\text { Risk Factors } \\
\text { One or more contraindications to outpatient treatment }\end{array}$} & \multicolumn{2}{|c|}{$\begin{array}{l}\text { Number of Outpatients } \\
(\%)\end{array}$} & \multicolumn{2}{|c|}{$\begin{array}{l}\text { Number of Inpatients } \\
(\%)\end{array}$} & \multirow{2}{*}{$\begin{array}{c}\begin{array}{c}\text { Hospitalization } \\
\text { rate }(\%)\end{array} \\
(79.1)\end{array}$} \\
\hline & 73 & $(7.0)$ & 277 & $(32.8)$ & \\
\hline $\begin{array}{l}\text { No contraindications to outpatient treatment but presence of } \\
\text { one or more comorbid conditions not in the PSI, }{ }^{*} \text { or } \\
\text { one or more treatments before presentation, }{ }^{\dagger} \text { or } \\
\text { multilobar radiographic infiltrates }\end{array}$ & 456 & $(43.7)$ & 398 & $(47.1)$ & $(46.6)$ \\
\hline No identifiable risk factors for hospitalization other than PSI risk class & 515 & $(49.3)$ & 170 & $(20.1)$ & $(24.8)$ \\
\hline All low-risk patients & 1,044 & $(100)$ & 845 & $(100)$ & $(44.7)$ \\
\hline
\end{tabular}

${ }^{*}$ Comorbid conditions not in the PSI included cognitive impairment, history of coronary artery disease, pulmonary disease, and diabetes mellitus.

${ }^{\dagger}$ Treatment before presentation included home oxygen therapy, oral or inhaled corticosteroid therapy, and antibiotic therapy within 7 days of presentation. 
cations for hospitalization and none have independent associations with increased mortality after controlling for other demographic and clinical characteristics found in the PSI. Further study is needed to determine whether physicians' reluctance to treat these patients in the outpatient setting is justified.

The limitations of our study should be acknowledged. First, demographic differences between patients who were and were not enrolled in the EDCAP trial may diminish the generalizability of our findings. However, the $80 \%$ enrollment rate was laudable for a large multicenter trial, and most of the nonenrolled patients were likely to be higher-risk patients and therefore excluded from the present study. Second, our analyses focused on patient, provider, and site characteristics available from retrospective chart review and physicians were not asked for the reason for hospital admission of low-risk patients. Future prospective studies are needed to document the physician reasons for hospital admission of low-risk patients with pneumonia. Third, the associations between the site of treatment and the characteristics examined in our study might have been altered by the design of the EDCAP trial. Although this trial was designed to test the effectiveness of 3 interventions of incremental intensity in increasing the proportion of low-risk patients treated as outpatients, ${ }^{11}$ we did not observe any statistically significant first-order interactions between intervention arm and patient, physician, and emergency department characteristics, suggesting that the associations found in our study were independent of the intervention arm. Fourth, the EDCAP trial was conducted in emergency departments in Connecticut and Western Pennsylvania and our findings may not extend to patients managed in other clinical settings or geographic locations.

In conclusion, in this trial to promote outpatient treatment of low-risk patients based on PSI risk class and level of arterial oxygenation, hospital admission was justified for onethird of low-risk inpatients based upon the presence of one or more predefined contraindications to outpatient treatment. At least one-fifth of low-risk inpatients who were hospitalized did not have a contraindication to outpatient treatment or an identifiable risk factor for hospitalization, suggesting that treating an even larger proportion of low-risk patients in the outpatient setting could be achieved without adversely affecting patient outcomes. The treatment of patients with no contraindications to outpatient treatment that have 1 or more risk factors for hospitalization is uncertain and deserves further study.

This research was conducted as part of the project, Guideline to Improve Quality of Initial Pneumonia Care, funded by the Agency for Healthcare Research and Quality (grant number R01 HS10049). Dr. M. J. Fine was supported in part by a K-24 career development award from the National Institute of Allergy and Infectious Diseases (5K24 Al01769). Dr. J. Labarere was supported by a grant from the Egide foundation, Paris, France (Programme Lavoisier), and by the Grenoble University Hospital (DRC, CHU Grenoble).

\section{REFERENCES}

1. Defrances CJ, Hall MJ, Podgornik MN. 2003 National Hospital Discharge Survey. Advance Data from Vital and Health Statistics of the Centers for Disease Control and Prevention. 2005:1-20.
2. Lave JR, Lin CCJ, Fine MJ, Hughes-Cromwick P. The cost of treating patients with community-acquired pneumonia. Semin Respir Crit Care Med. 1999;20:189-97.

3. Fine MJ, Hough LJ, Medsger AR, et al. The hospital admission decision for patients with community-acquired pneumonia. Results from the pneumonia patient outcomes research team cohort study. Arch Intern Med. 1997;157:36-44.

4. Coley CM, Li YH, Medsger AR, et al. Preferences for home vs hospital care among low-risk patients with community-acquired pneumonia. Arch Intern Med. 1996;156:1565-71.

5. Fine MJ, Auble TE, Yealy DM, et al. A prediction rule to identify low-risk patients with community-acquired pneumonia. N Engl J Med. 1997; 336:243-50.

6. Yealy DM, Auble TE, Stone RA, et al. Effect of increasing the intensity of implementing pneumonia guidelines: a randomized, controlled trial. Ann Intern Med. 2005; 143:881-94.

7. Marrie TJ, Lau CY, Wheeler SL, Wong CJ, Vandervoort MK, Feagan BG. A controlled trial of a critical pathway for treatment of communityacquired pneumonia. CAPITAL study investigators. Communityacquired pneumonia intervention trial assessing Levofloxacin. JAMA. 2000;283:749-55

8. Atlas SJ, Benzer TI, Borowsky LH, et al. Safely increasing the proportion of patients with community-acquired pneumonia treated as outpatients: an interventional trial. Arch Intern Med. 1998; 158:1350-6.

9. Marrie TJ, Huang JQ. Low-risk patients admitted with communityacquired pneumonia. Am J Med. 2005;118:1357-63.

10. Halm EA, Atlas SJ, Borowsky LH, et al. Understanding physician adherence with a pneumonia practice guideline: effects of patient, system, and physician factors. Arch Intern Med. 2000;160:98-104.

11. Yealy DM, Auble TE, Stone RA, et al. The emergency department community-acquired pneumonia trial: methodology of a quality improvement intervention. Ann Emerg Med. 2004;43:770-82.

12. Rice N. Binomial regression. In: Leyland $A H$, Goldstein $H$, eds. Multilevel Modelling of Health Statistics. Hoboken, NJ: John Wiley \& Sons Ltd; 2001:27-43.

13. Browne WJ. MCMC Estimation in MLwiN, Version 2.0. London, UK: Institute of Education, University of London; 2003.

14. Bartlett JG, Dowell SF, Mandell LA, File TM Jr, Musher DM, Fine MJ. Practice guidelines for the management of community-acquired pneumonia in adults. Infectious Diseases Society of America. Clin Infect Dis. 2000;31:347-82.

15. Mandell LA, Marrie TJ, Grossman RF, Chow AW, Hyland RH. Canadian guidelines for the initial management of community-acquired pneumonia: an evidence-based update by the Canadian Infectious Diseases Society and the Canadian Thoracic Society. The Canadian Community-Acquired Pneumonia Working Group. Clin Infect Dis. 2000; 31:383-421.

16. Mandell LA, Bartlett JG, Dowell SF, File TM Jr, Musher DM, Whitney C. Update of practice guidelines for the management of communityacquired pneumonia in immunocompetent adults. Clin Infect Dis. 2003;37:1405-33.

17. Goss CH, Rubenfeld GD, Park DR, Sherbin VL, Goodman MS, Root RK. Cost and incidence of social comorbidities in low-risk patients with community-acquired pneumonia admitted to a public hospital. Chest. 2003; 124:2148-55.

18. Arnold FW, Ramirez JA, McDonald LC, Xia EL. Hospitalization for community-acquired pneumonia: the pneumonia severity index vs clinical judgment. Chest. 2003;124:121-4.

19. Marras TK, Gutierrez C, Chan CK. Applying a prediction rule to identify low-risk patients with community-acquired pneumonia. Chest. 2000; 118:1339-43.

20. Stelianides S, Golmard JL, Carbon C, Fantin B. Influence of socioeconomic status on features and outcome of community-acquired pneumonia. Eur J Clin Microbiol Infect Dis. 1999;18:704-8.

21. Roson B, Carratala J, Dorca J, Casanova A, Manresa F, Gudiol F. Etiology, reasons for hospitalization, risk classes, and outcomes of community-acquired pneumonia in patients hospitalized on the basis of conventional admission criteria. Clin Infect Dis. 2001;33:158-65.

22. Metlay JP, Fine MJ. Testing strategies in the initial management of patients with community-acquired pneumonia. Ann Intern Med. 2003; 138:109-18.

23. Fine MJ, Smith MA, Carson CA, et al. Prognosis and outcomes of patients with community-acquired pneumonia. A meta-analysis. JAMA. 1996;275:134-41. 
24. Hasley PB, Albaum MN, Li YH, et al. Do pulmonary radiographic findings at presentation predict mortality in patients with community-acquired pneumonia? Arch Intern Med. 1996;156:2206-12.

\section{Supplementary Material}

The following supplementary material is available for this article online at www.blackwell-synergy.com
25. Carratala J, Fernandez-Sabe N, Ortega L, et al. Outpatient care compared with hospitalization for community-acquired pneumonia: a randomized trial in low-risk patients. Ann Intern Med. 2005; 142:165-72.

\section{Appendix}

